\title{
Rectal mobilization for laparoscopic pelvic lymphadenectomy of the lower paracervical pathway in patients with uterine cancer
}

\author{
Iori Kisu, MD, PhD ${ }^{1,2, *}$, Hidetaka Nomura, MD, PhD ${ }^{3, *}$, Miho lida, MD, PhD², Kouji Banno, MD, PhD², \\ Tetsuro Shiraishi, MD¹, Moito lijima, MD, PhD¹, Kayoko Nakamura, MD, PhD¹, Kiyoko Matsuda, MD, PhD', \\ Nobumaru Hirao, MD, PhD ${ }^{1}$

\begin{abstract}
Department of Obstetrics and Gynecology, ${ }^{1}$ Federation of National Public Service Personnel Mutual Aid Associations, Tachikawa Hospital, ${ }^{2}$ Keio University School of Medicine, ${ }^{3}$ Department of Gynecologic Oncology, Cancer Institute Hospital, Tokyo, Japan
\end{abstract}

\section{Objective}

The pelvic lymphatic drainage system comprises the upper and lower paracervical pathways (LPPs). Lymph node dissection of the LPP, including the cardinal ligament, internal iliac, internal common iliac, and presacral lymph nodes, requires higher surgical skills because of the anatomical limitations of the pelvic cavity and the dissection of vessels while preserving the nerves in the pelvic floor. In this video, we demonstrate rectal mobilization for laparoscopic complete pelvic lymph node dissection of the LPP in patients with uterine cancer.

\section{Methods}

Rectal mobilization was performed before complete pelvic lymph node dissection of the LPP. The pararectal space was opened widely and the connective tissue between the presacral fascia and prehypogastric nerve fascia was dissected bilaterally, allowing the rectum to be pulled.

Results

This procedure created a wide-open space in the pelvic floor, allowing clear visualization of the nerves and lymph nodes of the LPP. Laparoscopic complete lymph node dissection of the LPP was performed in the open space while preserving the hypogastric and pelvic splanchnic nerves and isolating the extensive network of blood vessels in the pelvic cavity.

\section{Conclusion}

Rectal mobilization enabled the safe execution of laparoscopic complete pelvic lymph node dissection of the LPP in patients with uterine cancer.

Keywords: Cervical cancer; Laparoscopic surgery; Pelvic lymphatic pathway; Uterine cancer

Systematic lymphadenectomy is a potential indicator of surgical quality for the staging and prognosis of gynecological cancers. The pelvic lymphatic drainage system comprises the upper paracervical pathway and lower paracervical pathway (LPP) (Fig. 1) [1,2]. The lymph nodes along the LPP include the cardinal ligament, internal iliac, internal common iliac, and presacral lymph nodes. Paracervical lymphadenectomy of the LPP, defined as the removal of the lymph node tissues below the obturator nerve and around the deep uterine vein [3], is the key step in radical hysterectomy for detecting parametrial lymph nodes in early-stage cervical cancer [4]. A thor-
Received: 2021.06.15. Revised: 2021.08.11. Accepted: 2021.08.11. Corresponding author: Iori Kisu, $\mathrm{MD}, \mathrm{PhD}$

Department of Obstetrics and Gynecology, Federation of National Public Service Personnel Mutual Aid Associations, Tachikawa Hospital, 4-2-22 Nishiki, Tachikawa, Tokyo 1908531, Japan E-mail: iori71march@hotmail.co.jp https://orcid.org/0000-0003-1143-9855

*These authors contributed equally to this work.

Articles published in Obstet Gynecol Sci are open-access, distributed under the terms of the Creative Commons Attribution Non-Commercial License (http://creativecommons. org/licenses/by-nc/3.0/) which permits unrestricted non-commercial use, distribution, and reproduction in any medium, provided the original work is properly cited.

Copyright $\odot 2021$ Korean Society of Obstetrics and Gynecology 


\section{Obstetrics \& Gynecology Science}

Vol. 64, No. 6, 2021

ough dissection of the parametrial lymph nodes improves the detection rate of parametrial lymph node metastasis [5]. Therefore, the creation of wide paravesical and pararectal spaces by the suspension of the rectum and umbilical liga-

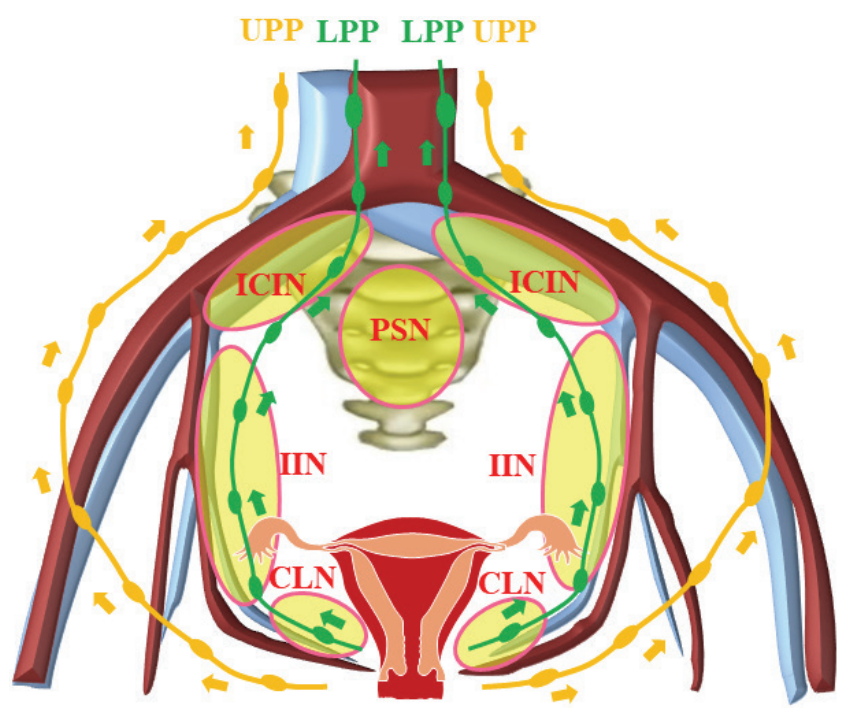

Fig. 1. Schema of the pelvic lymphatic drainage pathways from the uterus. The pelvic lymphatic drainage system from the uterus comprises the upper (yellow arrows) and lower (green arrows) paracervical pathways. The lower paracervical pathway includes the cardinal ligament, internal iliac, presacral, and internal common iliac nodes. UPP, upper paracervical pathway; LPP, lower paracervical pathway; ICIN, internal common iliac nodes; PSN, presacral nodes; IIN, internal iliac nodes; CLN, cardinal ligament nodes. ment without direct handling of the uterine cervix is essential for sufficient resection of the parametrium in laparoscopic radical hysterectomy for cervical cancer [6]. Moreover, onethird of patients with endometrial cancer who are lymph node-positive have metastases along the LPP $[1,7]$.

The extent of lymphadenectomy often reflects the surgeons' preferences based on their personal experiences [7]. Lymph node dissection of the LPP requires higher surgical skills because it is performed in the deep and narrow pelvic space. Furthermore, the procedure is limited anatomically by the pelvic cavity due to the central presence of the rectum. Thus, it is difficult to secure a space and dissect the vessels while preserving the nerves on the pelvic floor (Fig. 2). Therefore, surgical approaches are needed that allow complete pelvic lymph node dissection of the LPP.

Because the pelvic fascia, nerves around the rectum in the pelvic floor, and lymph nodes of the LPP need to be dissected in this limited space, particular attention should be given to preserving the hypogastric and inferior hypogastric plexus attached to the prehypogastric nerve fascia. It is also important to avoid damaging the vascular structures, such as the internal iliac vessels and deep uterine veins (Fig. 2). To address these problems, a surgical technique involving rectal mobilization was developed for laparoscopic pelvic lymph node resection of the LPP. This study demonstrated this rectal mobilization for laparoscopic complete pelvic lymph node dissection of the LPP in patients with uterine cancer.

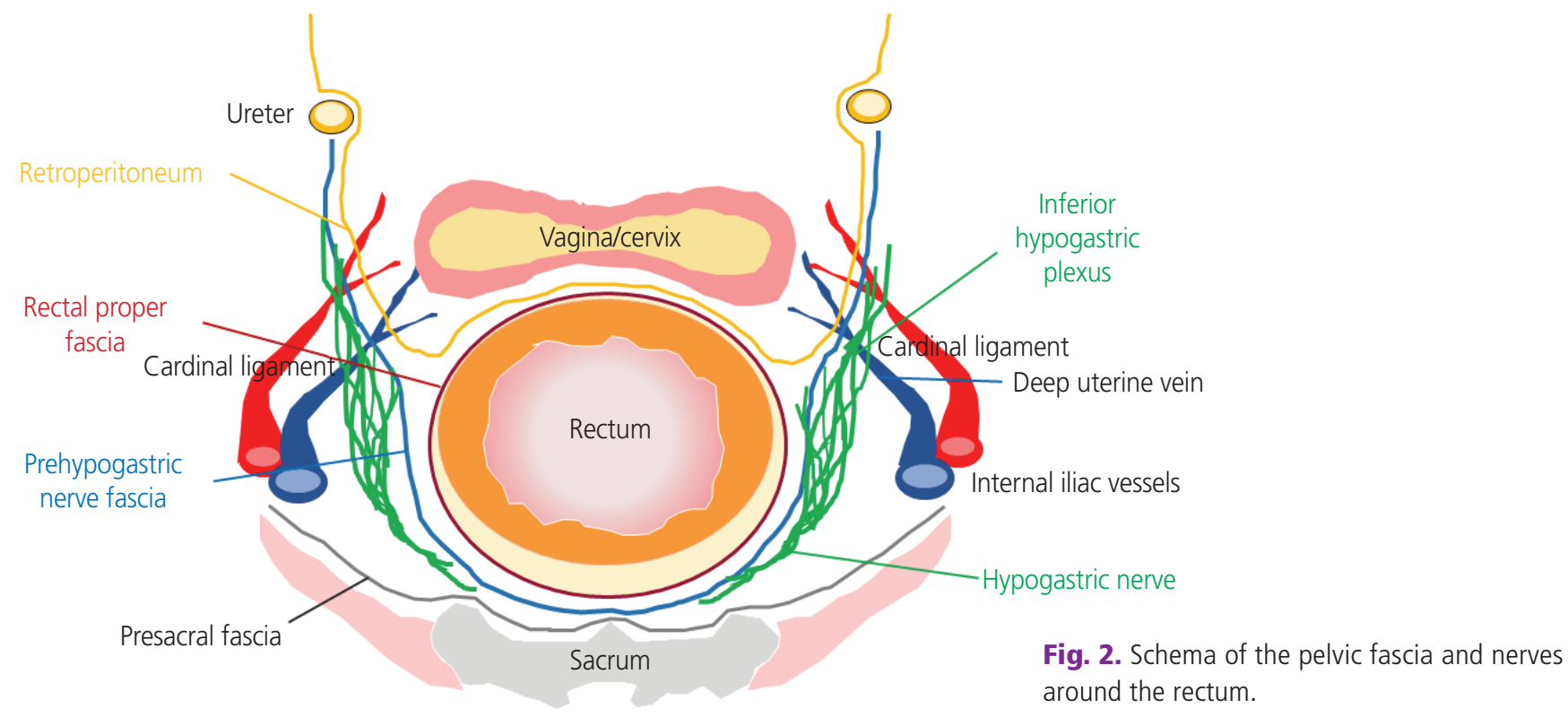




\section{Obstetrics \& Gynecology Science}

Iori Kisu, et al. Rectal mobilization for lymphadenectomy
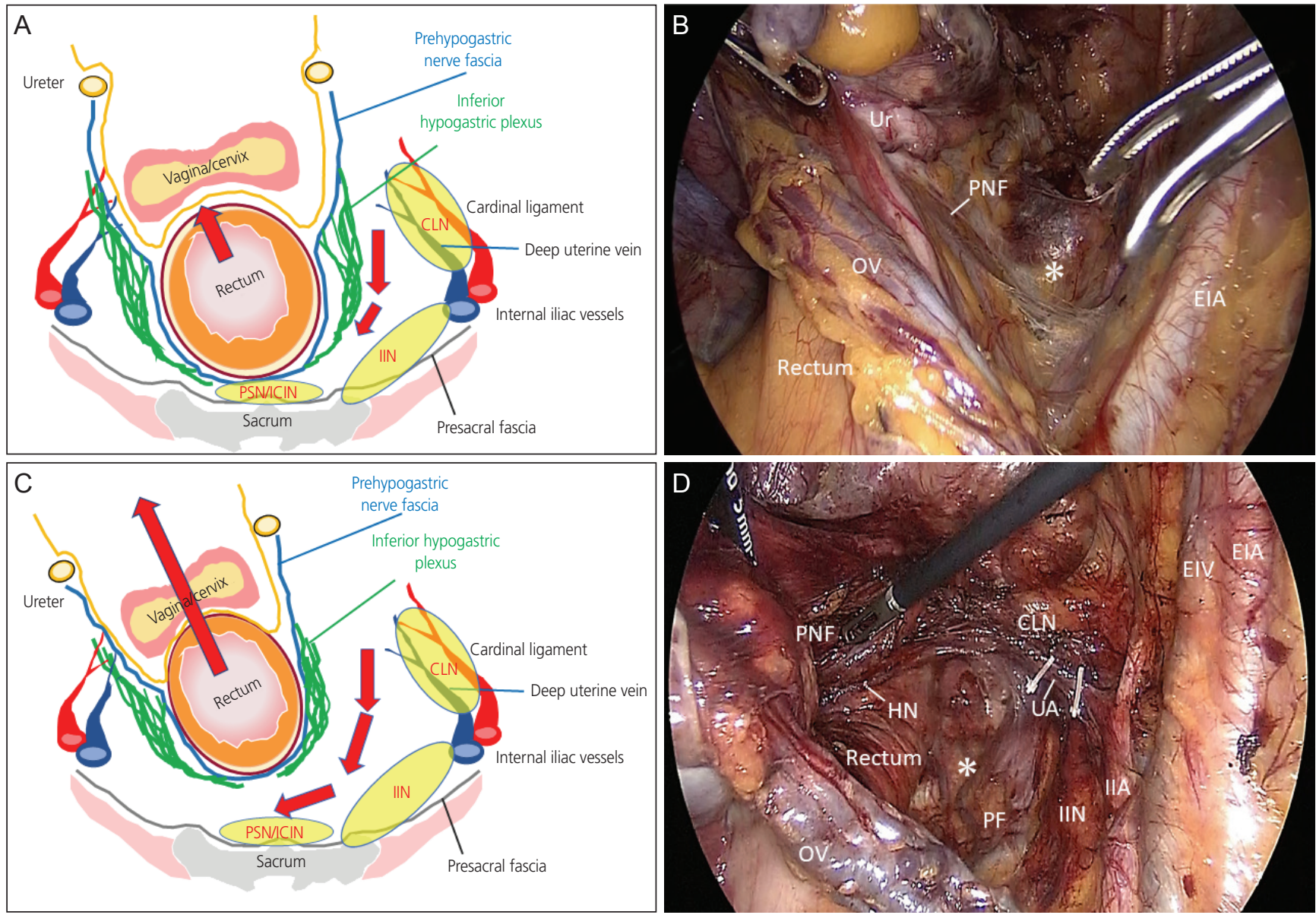

Fig. 3. Rectal mobilization for pelvic lymph node dissection of the lower paracervical pathway. (A) The rectum is slowly mobilized by opening the retroperitoneal and Latzko's pararectal spaces between internal iliac vessels and prehypogastric nerve fascia. (B) Intraoperative image after dissection of the retroperitoneal and Latzko's pararectal spaces $\left(^{*}\right)$. (C) The rectum is moved ventrally after further dissection of the pararectal space between the presacral fascia and prehypogastric nerve fascia. (D) Intraoperative image after dissection of the pararectal space $\left({ }^{*}\right)$ between the presacral fascia and prehypogastric nerve fascia. CLN, cardinal ligament nodes; IIN, internal iliac nodes; PSN, presacral nodes; ICIN, internal common iliac nodes; Ur, ureter; PNF, prehypogastric nerve fascia; OV, ovarian vein; EIA, external iliac artery; EIV, external iliac vein; HN, hypogastric nerve; UA, uterine artery; PF, presacral fascia; IIA, internal iliac artery.

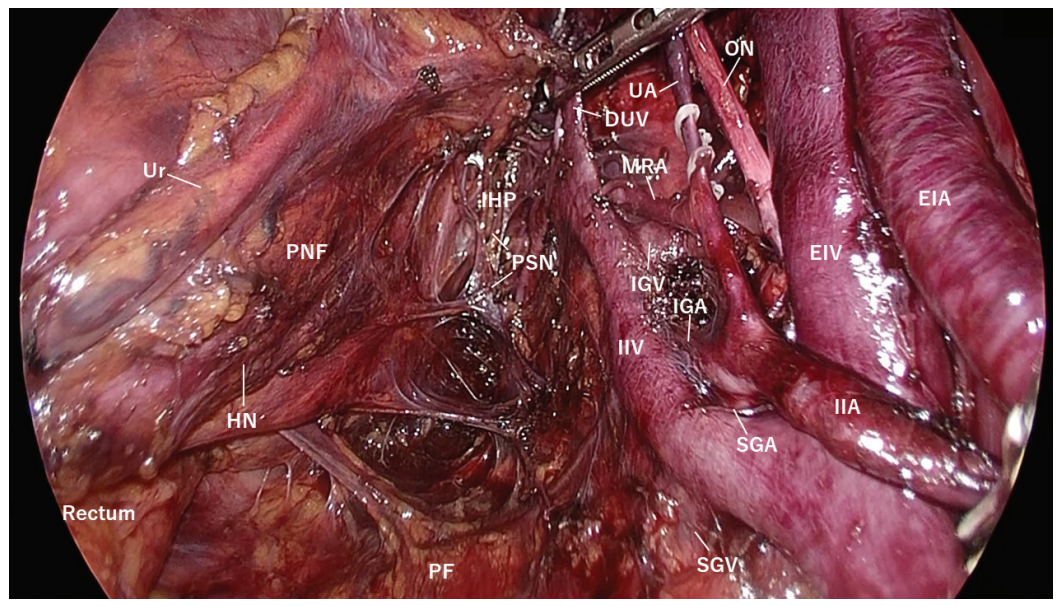

Fig. 4. Intraoperative image of the right lower paracervical pathway. The large pararectal space created by rectal mobilization allows complete dissection of the pelvic lymph nodes while preserving the nerve systems and isolating the extensive network of blood vessels in the pelvic cavity. ON, obturator nerve; UA, uterine artery; DUV, deep uterine vein; Ur, ureter; IHP, inferior hypogastric plexus; MRA, middle rectal artery; EIA, external iliac artery; PNF, prehypogastric nerve fascia; PSN, pelvic splanchnic nerve; IGV, inferior gluteal vein; IGA, inferior gluteal artery; EIV, external iliac vein; IIV, internal iliac vein; $H N$, hypogastric nerve; SGA, superior gluteal artery; IA, internal iliac artery; PF, presacral fascia; SGV, superior gluteal vein. 


\section{Obstetrics \& Gynecology Science}

Vol. 64, No. 6, 2021

Rectal mobilization was performed before complete laparoscopic lymph node dissection. After cutting the round ligament, the retroperitoneal and Latzko's pararectal spaces were widely opened between the internal iliac vessels and prehypogastric nerve fascia. The rectum was gradually mobilized by pulling it upwards (Fig. 3A, B). Subsequently, the connective tissue between the presacral and prehypogastric nerve fascia was dissected bilaterally, allowing ventral elevation of the rectum. These procedures created an open space in the pelvic floor, allowing visualization of the hypogastric nerve, pelvic splanchnic nerve, inferior hypogastric plexus, and lymph nodes of the LPP (Fig. 3C, D). Complete lymph node dissection of the LPP was then performed.

By exerting upward traction on the rectosigmoid with a thread through a mesh wrapped around the rectum, the internal common iliac lymph nodes and presacral lymph nodes were revealed while identifying the ureter medially. Complete removal of these lymph nodes was then performed after exposing the sacral promontory with caution to avoid damaging the common iliac and median sacral veins. The large pararectal space created by rectal mobilization allowed adequate vascular isolation in the internal iliac and cardinal ligament areas. The internal iliac and cardinal lymph nodes were safely removed while isolating the extensive network of blood vessels in the pelvic cavity and preserving the hypogastric and pelvic splanchnic nerves and inferior hypogastric plexus (Fig. 4).

In conclusion, rectal mobilization created a large open space in the pelvic cavity, allowing the safe execution of laparoscopic complete pelvic lymph node dissection of the LPP and extensive vessel dissection while preserving the nerves of the pelvic floor in patients with uterine cancer.

\section{Conflict of interest}

No potential conflict of interest relevant to this article was reported.

\section{Ethical approval}

This study was approved by the Institutional Review Board (IRB) of the Federation of National Public Service Personnel Mutual Aid Associations, Tachikawa hospital (IRB No. 2020-
10), and written informed consent was obtained from all patients. The study was performed in accordance with the principles of the Declaration of Helsinki.

\section{Patient consent}

The patient provided written informed consent for the publication of this manuscript and related video.

\section{Funding information}

None.

\section{Video clip}

Video can be found with this article online at https://doi. org/10.5468/ogs.21190.

\section{References}

1. Geppert B, Lönnerfors C, Bollino M, Arechvo A, Persson J. A study on uterine lymphatic anatomy for standardization of pelvic sentinel lymph node detection in endometrial cancer. Gynecol Oncol 2017;145:256-61.

2. Persson J, Geppert B, Lönnerfors C, Bollino M, Måsbäck $A$. Description of a reproducible anatomically based surgical algorithm for detection of pelvic sentinel lymph nodes in endometrial cancer. Gynecol Oncol 2017;147:120-5.

3. Querleu D, Morrow CP. Classification of radical hysterectomy. Lancet Oncol 2008;9:297-303.

4. Van den Tillaart SA, Trimbos JB, Dreef EJ, Jordanova ES, Fleuren GJ. Patterns of parametrial involvement in radical hysterectomy specimens of cervical cancer patients. Int J Gynecol Pathol 2011;30:185-92.

5. Zhao D, Li B, Zheng S, Ou Z, Zhang Y, Wang Y, et al. Separate lateral parametrial lymph node dissection improves detection rate of parametrial lymph node metastasis in early-stage cervical cancer: 10-year clinical evaluation in a single center in China. Chin J Cancer Res 2020;32:804-14. 


\section{Obstetrics \& Gynecology Science}

lori Kisu, et al. Rectal mobilization for lymphadenectomy

6. Kanao H, Matsuo K, Aoki Y, Tanigawa T, Nomura H, Okamoto $\mathrm{S}$, et al. Feasibility and outcome of total laparoscopic radical hysterectomy with no-look no-touch technique for FIGO IB1 cervical cancer. J Gynecol Oncol 2019;30:e71.

7. Odagiri T, Watari H, Kato T, Mitamura T, Hosaka M,
Sudo S, et al. Distribution of lymph node metastasis sites in endometrial cancer undergoing systematic pelvic and para-aortic lymphadenectomy: a proposal of optimal lymphadenectomy for future clinical trials. Ann Surg Oncol 2014;21:2755-61. 